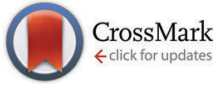

Cite this: Mater. Horiz., 2017, 4, 236

Received 7th November 2016, Accepted 16th December 2016

DOI: $10.1039 / c 6 m h 00495 d$

www.rsc.li/materials-horizons

\title{
Reversible transition between isotropic and anisotropic thermal transport in elastic polyurethane foams $\dagger$
}

\author{
M. Chau,,$^{a}$ B. A. F. Kopera, $\ddagger^{b}$ V. R. Machado, ${ }^{a}$ S. M. Tehrani, ${ }^{\text {ac }}$ M. A. Winnik, ${ }^{\text {ac }}$ \\ E. Kumacheva*acd and M. Retsch*b
}

\begin{abstract}
Elastic composite foams with anisotropic lamellar microstructures were formed by freeze-casting polyurethane dispersions with graphitic additives. This anisotropy resulted in orientation-dependent mechanical and thermal properties. Most importantly, the effective thermal conductivity can be reversibly transitioned between anisotropic and isotropic states by altering the thermal conductivity of the contained atmosphere.
\end{abstract}

The ability to control the flow of heat is an essential contribution towards an energy efficient use of our natural resources. Recent efforts have been devoted to access thermal switches, ${ }^{1,2}$ thermal rectifiers, ${ }^{3,4}$ and thermal insulators ${ }^{5,6}$ by intricate material selection and design. In particular, for thermal insulation applications, porous materials represent the most highly investigated class of materials. Nevertheless, most foams are isotropic materials leaving the orientation-dependent thermal properties unoptimized. Future insulation materials will, however, not only need to control the flow of heat along a particular direction, but will also be required to be dynamically tunable. ${ }^{7}$ Such dynamic insulation materials, could prevent heat loss and promote heat recovery by utilizing anisotropic open-cell foams that are thermally insulating in the orientation perpendicular to a wall, while thermally conductive in the parallel direction. Therefore, we introduce polyurethane foams with a strongly anisotropic microcellular structure. Most importantly, we demonstrate the general guidelines to reversibly control the thermal transport in such foams between isotropic and anisotropic states. This paves the way towards future intelligent thermal insulation materials.

\footnotetext{
${ }^{a}$ Department of Chemistry, University of Toronto, 80 Saint George Street, Toronto, Ontario, M5S 3H6, Canada. E-mail: ekumache@chem.utoronto.ca

${ }^{b}$ University of Bayreuth, Physical Chemistry - Polymer Systems, Universitätsstraße 30, 95447 Bayreuth, Germany. E-mail: markus.retsch@uni-bayreuth.d

${ }^{c}$ University of Toronto, Department of Chemical Engineering and Applied Chemistry, 200 College Street, Toronto, Ontario M5S 3E5, Canada

${ }^{d}$ University of Toronto, The Institute of Biomaterials and Biomedical Engineering, 4 Taddle Creek Road, Toronto, Ontario M5S 3G9, Canada

$\dagger$ Electronic supplementary information (ESI) available. See DOI: 10.1039/c6mh00495d \$ Authors contributed equally.
}

\begin{abstract}
Conceptual insights
We present a new concept in the design of dynamic insulation materials with external control over the magnitude and orientation of the thermal conductivity within a porous material. We use thermal conductivity matching to realize two states of thermal transport in polyurethane foam: isotropic and anisotropic. Our system is based on an anisotropic foam skeleton comprising polyurethane and a co-continuous gas phase. The thermal conductivity of the gas phase can be adjusted using its pressure and its composition. Choosing the right conditions leads to a match of the thermal conductivities of the gas and the polyurethane phase. This allows the effective thermal transport properties of the foam to be reversibly changed both in magnitude and orientation within seconds. The thermal conductivity matching concept of our study is expected to be applicable to other biphasic, co-continuous systems as well. Therefore, we provide a general concept for future dynamic insulation materials. Our foams are accessible via a particularly simple method: freeze-casting of a polyurethane dispersion. We show that the unique thermal transport properties are combined with good mechanical stability and recoverability.
\end{abstract}

State-of-the-art thermal insulation is achieved by highly porous and consequently lightweight materials. ${ }^{8}$ Thereby, silica aerogels resemble the most efficient class of materials, with thermal conductivities reaching $<30 \mathrm{~mW} \mathrm{~m}{ }^{-1} \mathrm{~K}^{-1} \cdot{ }^{9,10}$ However, the fabrication of highly porous silica aerogels requires in most cases the use of supercritical drying. ${ }^{11}$ Additionally, the resulting porous monoliths are rather brittle. ${ }^{12}$ These shortcomings contributed to a limited application of aerogel materials until now, and only recently aerogel products have been commercialised, e.g. by САВОТ. Much more widespread are polymer-based foams, which reach thermal conductivities as low as $17 \mathrm{~mW} \mathrm{~m}{ }^{-1} \mathrm{~K}^{-1}$ (e.g. SLENTITE ${ }^{\circledR}$ from BASF).

Polymer foams combine the inherent low thermal conductivity of the bulk polymer materials with a low density. Yet, their viscoelastic properties render them more resilient to mechanical fatigue compared to brittle aerogels. Polymer foams can be classified into closed- and open-celled structures, depending on their cell morphology. The most widespread and ubiquitous examples are expanded polystyrene, a rigid, closed cell foam, and polyurethane foams, which are elastic and open cell. Commercial polymer foams 
are fabricated via batch foaming, extrusion foaming or injection molding; these processes result in pore sizes in the range of tens and hundreds of micrometers. ${ }^{13}$

The introduction of nanosized pores strongly reduces the overall thermal conductivity of a polymer foam. This is caused by the Knudsen effect, which becomes dominant when the cell size is on the same order of magnitude as the mean free path of the internal gas phase (typically tens of $\mathrm{nm}$ ) ${ }^{14,15}$ In this regime, a reduction in cell size reduces the effective thermal conductivity. If the morphology remains isotropic, the thermal transport properties alter equivalently with the pore size, regardless of the foam orientation.

A method that has recently evolved to access highly anisotropic porous materials is freeze-casting. Here, a temperature gradient is applied to direct the growth of ice crystals. Concomitantly, any dispersed or dissolved material in the aqueous phase is rejected from the growing ice crystals. After freeze-drying an oriented network of the dispersed material, which corralled the ice crystals, is retained. ${ }^{16}$ Freeze-casting itself has evolved into a vital field of research, where remarkable structural control can now be achieved ${ }^{17,18}$ Freeze-casting of latex dispersions is characterized by the evolution of three zones of varying morphologies, where lamellar and dendritic growth dominate. ${ }^{19,20}$ Typical lamellae thicknesses and spacings obtained by using this method are on the order of several micrometers.

Freeze-casting has been used to access insulating foams with anisotropic thermal transport properties. Hostler et al. achieved a ratio of about 1.3 between thermal transport along and perpendicular to the foam matrix consisting of a clay sheet. ${ }^{21}$ In a recent work, Wicklein $e t$ al. demonstrated multifunctional properties of anisotropically porous materials, which were accessed through freeze-casting. ${ }^{5}$ Using a combination of cellulose nanofibers, graphene oxide, and sepiolite nanorods, they obtained a rigid, open-celled foam, with a very low radial thermal conductivity of only $\sim 15 \mathrm{~mW} \mathrm{~m}^{-1} \mathrm{~K}^{-1}$ and a much higher thermal conductivity along the lamellae $\left(\sim 170 \mathrm{~mW} \mathrm{~m}{ }^{-1} \mathrm{~K}^{-1}\right)$. At the same time, these foams possessed good fire-retardant properties.

In order to elucidate the potential of anisotropic foams to function as a future dynamic insulation material, we report here the freeze-casting of polyurethane (PU) dispersions into elastomeric foams. The resulting highly anisotropic and lamellar structures lead to orientation-dependent thermal conductivities and Young's moduli. Most importantly, we demonstrate how the orientation-dependent thermal transport properties can be specifically turned "on" and "off". Using several additive materials in the polymer matrix, we can further deduce the general design rules to obtain such dynamically tunable materials.

The fabrication of anisotropic foams involves five steps: synthesizing a PU dispersion, dispersing the additives (if necessary), freeze-casting, freeze-drying, and thermal annealing of the foam. The PU was designed to yield minimum crystallinity and to allow for the generation of water-stable PU nanoparticles. Therefore, a block-copolymer polyol (polycaprolactone-block-polytetrahydrofuranblock-polycaproclatone), dimethylol propionic acid, and isophorone diisocyanate were used to synthesize the PU prepolymer (the details are given in the ESI, $\uparrow$ Scheme S1 and Fig. S1, S2).
This polymer was transformed into colloidally stable nanoparticles via nanoprecipitation. The particle size was determined to be $14 \pm 4 \mathrm{~nm}$ by cryo-TEM (Fig. S3, ESI $\dagger$ ) with an electrokinetic potential of $-52 \pm 2 \mathrm{mV}$. The PU dispersion was freeze-cast into anisotropic PU foams (Fig. S4, ESI $\dagger$ ). The temperature of the bottom of the mold was set to $-20{ }^{\circ} \mathrm{C}$ to prevent crystallization of the polyester and polyether components of the PU. After freeze-drying, the $\mathrm{PU}$ foam (termed $\mathrm{PU}_{\text {pure }}$ further on) was thermally annealed at $90{ }^{\circ} \mathrm{C}$ for eight hours to promote crosslinking and hydrogen bonding between the PU segments, as well as to reduce the tackiness of the foam surface.

The morphology of the freeze-cast foam is summarized in Fig. 1. Its overall size and form is given by the mold inner dimensions and is retained throughout the templating procedure (Fig. 1a). The small PU nanoparticles are fused together into PU lamellae during ice-crystal growth and thermal annealing, resulting in a continuous polymer matrix. This polymer matrix is highly anisotropic as can be inferred from the photograph, the microcomputed tomography (microCT) image, and the scanning electron microscopy (SEM) images (Fig. 1a-d). Fig. 1b demonstrates that a preferential orientation is imposed only along the direction of ice growth, with large pores, which are at least $930 \mu \mathrm{m}$ long and have an aspect ratio $>31$. We term this axis as the "parallel" axis, throughout this work. The axes orthogonal to the direction of
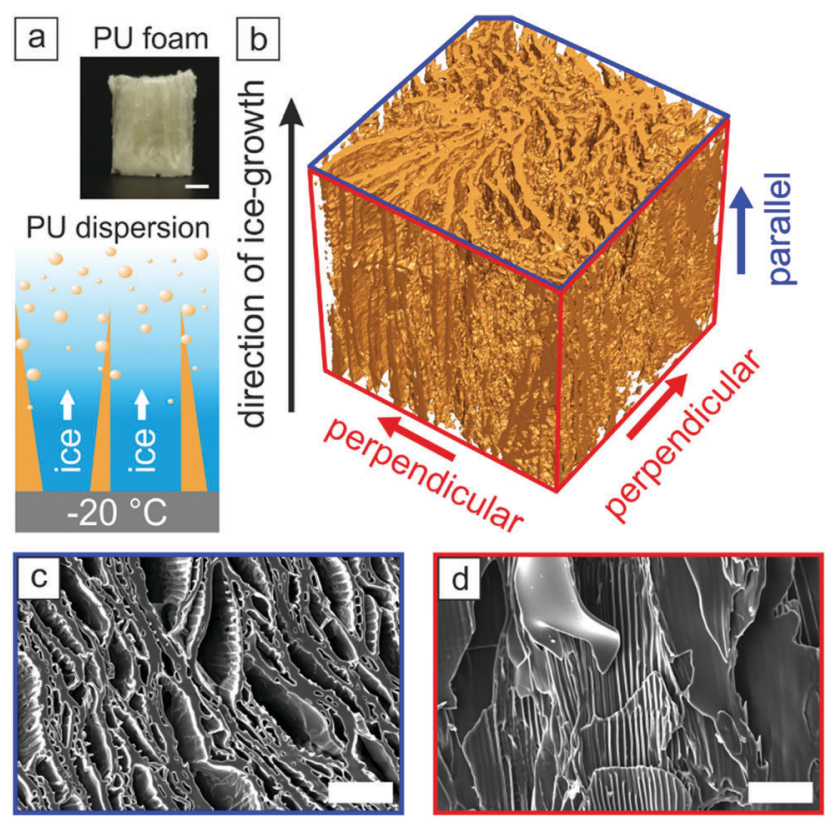

Fig. 1 (a) Top: Photograph of the $\mathrm{PU}_{\text {pure }}$ foam after freeze-drying and thermal annealing (scale bar: $2.5 \mathrm{~mm}$ ). Even macroscopically, the upright orientation of the lamellae can be inferred. Bottom: Schematic representation of the lamellae formation during the ice-crystal growth in an aqueous dispersion. (b) Microtomography image of a $\mathrm{PU}_{\text {pure }}$ foam. The length of the cube is $930 \mu \mathrm{m}$. The lamellae are oriented along the ice growth direction without preferential orientation within the perpendicular planes. (c) SEM top-view image of the $P U_{\text {pure }}$ lamellae showing no preferential orientation perpendicular to the ice-growth direction (scale bar: $100 \mu \mathrm{m}$ ). (d) Side-view SEM image of the $P U_{\text {pure }}$ lamellae showing dendritic undulations on the lamellae surfaces and strong orientation along the direction of ice growth (scale bar: $100 \mu \mathrm{m}$ ). 
ice-growth are termed "perpendicular". As shown in the microCT image (Fig. $1 \mathrm{~b}$ and video in the ESI $\dagger$ ), these continuous lamellae are curvilinear, without any preferential alignment in the plane perpendicular to the ice-growth direction. The lamellar thicknesses were $\sim 5-10 \mu \mathrm{m}$ and their inter-lamellar distances are on the order of $10-30 \mu \mathrm{m}$ (Table S1, ESI $\dagger$ ), which is in accordance with the length scales reported in the literature. ${ }^{19}$ A closer inspection of Fig. 1d reveals the influence of dendritic ice, which templates into regularly spaced protrusions $(\sim 13 \mu \mathrm{m})$ on the sidewall of the PU lamellae (also highlighted in Fig. S5e, ESI $\dagger$ ). ${ }^{19}$ The freeze-casting process is robust and reliably results in pore sizes of $10-30 \mu \mathrm{m}$.

Differential scanning calorimetry (DSC, Fig. S6, ESI $\dagger$ ) and $\mathrm{X}$-ray diffraction (XRD, Fig. S7, ESI $\dagger$ ) measurements were used to characterize the polymer microstructure of the PU foam. Both measurements indicated the absence of crystalline domains and suggested that the PU was in an amorphous state at room temperature.

The thermal diffusivity of this foam was assessed by Xenon flash analysis (XFA). The foam monolith was cut along its perpendicular and parallel planes in order to obtain thin slabs, which allowed for the anisotropic thermal transport characterization.

The thermal diffusivity was then used to calculate the thermal conductivity, $\kappa$, using

$$
\kappa=\alpha \cdot c_{\mathrm{p}} \cdot \rho
$$

where the specific heat capacity, $c_{\mathrm{p}}$, of the PU matrix was obtained by DSC, and $\rho$ was the density of the foam. In vacuum, the thermal conductivity along the parallel orientation, $\kappa_{\text {para }}$, was measured to be $59 \pm 3 \mathrm{~mW} \mathrm{~m}^{-1} \mathrm{~K}^{-1}$, while in the perpendicular orientation, $\kappa_{\text {perp }}$, was only $11 \pm 1 \mathrm{~mW} \mathrm{~m}^{-1} \mathrm{~K}^{-1}$. In either direction, the thermal conductivities are significantly reduced compared to bulk PU $\left(\sim 200 \mathrm{~mW} \mathrm{~m}^{-1} \mathrm{~K}^{-1}\right),{ }^{22}$ due to their higher porosity.

For our system in vacuum, we found a ratio of parallel to perpendicular thermal conductivity of $\sim 5.4$. This can be qualitatively visualized when exciting a thin slab of this foam by a small heat source, in our case a focused laser beam. As can be seen in Fig. 2e, the temperature spreads isotropically when the lamellae are oriented parallel to the laser beam. However, upon perpendicular orientation the temperature distribution is anisotropic following the preferential orientation of the lamellae (horizontal in Fig. 2e).

The degree of anisotropy in these foams is less compared to those made by Wicklein et al. We attribute this difference to the lack of lateral orientational order, whereas a preferential orientation can be inferred within the perpendicular direction in their case. ${ }^{5}$ An even higher degree of anisotropy has been recently reported in bulk films for graphenoxide sheets blended with cellulose nanofibers. ${ }^{23}$ This highlights the strong influence of the orientational order on the anisotropic thermal conductivity. One should note, however, that we were able to achieve a substantial degree of anisotropy using fully isotropic objects, based on spherical PU nanoparticles.

The outstanding thermal behaviour of this foam is revealed, when conducting the Xenon flash analysis (XFA) measurements in helium (He). Under $\mathrm{He}$ at $1000 \mathrm{mbar}$, the anisotropic thermal conductivity vanishes and the thermal conductivity in
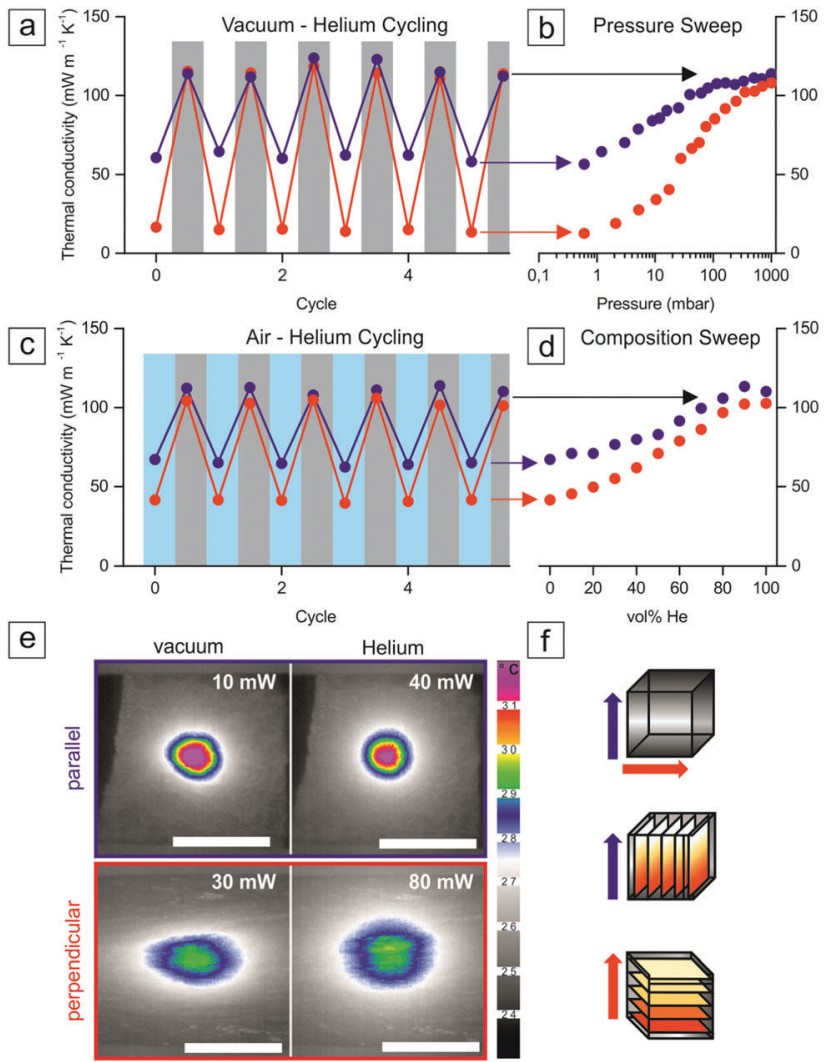

Fig. $2(a-d)$ Thermal conductivities of $P U_{\text {pure }}$ foams in the parallel (blue) and perpendicular (red) orientations. (a) States of anisotropic and isotropic thermal conductivity of $\mathrm{PU}_{\text {pure }}$ via cycling between vacuum ( $<1 \mathrm{mbar}$ ) and helium (1000 mbar). (b) Corresponding parallel (blue) and perpendicular (red) thermal conductivities at various pressures of helium. (c) Cycling between air and helium at a constant pressure of 980 mbar. (d) Corresponding parallel (blue) and perpendicular (red) thermal conductivities at various volume fractions of helium in a helium/air mixture. (e) Infrared thermograms of thin slabs of $\mathrm{PU}_{\text {pure }}$ irradiated by a small laser spot on the backside. The temperature distribution after $\sim 30 \mathrm{~s}$ of laser exposure indicates the anisotropic temperature spreading in a vacuum (left panels). In helium (right panels) the temperature distribution resumes a more isotropic behavior. The numbers in the top right corner indicate the nominal laser power used to achieve comparable temperatures. ( $f$ ) Cartoon of the orientation of the anisotropic PU foam used for the thermograms shown in (e).

both orientations increases to about $115 \mathrm{~mW} \mathrm{~m}^{-1} \mathrm{~K}^{-1}$. Note, that only conductive heat transport has to be considered in these foams since the small pore size inhibits convective contributions. We analysed this interesting effect in detail by measuring the pressure-dependent thermal conductivity along each orientation (Fig. 2b). The degree of anisotropy increases monotonically with decreasing He pressure. The reduction in thermal conductivity is more significant along the perpendicular than along the parallel direction. This can be rationalized in two ways. Firstly, heat can hardly spread across the foam in its perpendicular orientation in vacuum due to the low number of inter-lamellar contact points. This is a direct consequence of the anisotropic morphology of the foam. However, the presence of gas in the pores introduces a thermal transport pathway along the perpendicular orientation, which in the case of He can even match the thermal conductivity of the polymer skeleton. Secondly, the 
mean free path of the He gas phase depends strongly on the gas pressure: it increases significantly with the decreasing pressure. The thermal conductivity becomes pressure dependent once the mean free path is on the length scale of the confinement. Consequently, the He thermal conductivity drops once the gas mean free path is limited by the characteristic cell size. This effect is known as the Knudsen effect. ${ }^{14,24}$ The transition from the free gas regime to the Knudsen regime for He is shown in Fig. S13 (ESI $\dagger$ ). The pore dimensions are significantly smaller in the perpendicular orientation $(\sim 10-30 \mu \mathrm{m})$ than in the parallel orientation (up to $\mathrm{mm}$ ). Therefore, the thermal conductivity of the gas phase is more strongly reduced in the perpendicular than in the parallel orientation. As demonstrated in Fig. 2a, we were able to repeatedly and reversibly toggle between the states of isotropic and anisotropic thermal transport.

We further extended this concept of reversible thermal anisotropy by varying the effective thermal conductivity of the foam by varying the gas composition. We changed the composition of the atmosphere, at constant pressure ( $980 \mathrm{mbar}$ ), from $100 \%$ air $\left(26 \mathrm{~mW} \mathrm{~m}^{-1} \mathrm{~K}^{-1}\right)^{25}$ to $100 \% \mathrm{He}\left(155 \mathrm{~mW} \mathrm{~m}{ }^{-1} \mathrm{~K}^{-1}\right){ }^{26}$ Fig. 2 c. (Note that air is a bad thermal conductor, while He is a good thermal conductor.) With the increasing content of air, a higher degree of anisotropy was observed. However, the ratio of parallel to perpendicular thermal conductivity decreased to about 2 in pure air compared to 5 for the vacuum case. As explained before, this is a consequence of the presence of air in the porous voids, which allows for the transfer of heat between the lamellae.

Overall, our experiments show two pathways towards creating a dynamically tunable insulation system: via changing the gas pressure, and via changing the gas composition inside the foam. Both methods can be externally controlled in an open porous structure by adjusting the surrounding atmosphere.

In order to deduce the general design criteria for fabricating dynamically tunable foams, we explored how the properties of the foam skeleton affected the thermal anisotropy. Therefore, we fabricated three composite foams, in which cellulose nanocrystals (CNC), carbon black (CB), and carbon nanofibers (CNF) were introduced into the PU dispersion (the details are given in the ESI, $†$ Fig. S8-S11). Upon freeze-casting the dispersion, the additives were incorporated into the lamellae. CNC was chosen as it aids the dispersion of the graphitic components without further functionalization (Fig. S10, ESI $\dagger$ ). ${ }^{23,28}$ Graphitic additives were used to significantly increase the thermal conductivity of the skeleton. The resulting foams were consequently termed $\mathrm{PU}_{\mathrm{CNC}}, \mathrm{PU}_{\mathrm{CNC}-\mathrm{CB}}$, and $\mathrm{PU}_{\mathrm{CNC}-\mathrm{CNF}}$, respectively.

The structures of the composite PU foams were characterised using SEM (Fig. S5, S11 and Table S1, ESI $\dagger$ ). No significant changes compared to $\mathrm{PU}_{\text {pure }}$ could be detected for $\mathrm{PU}_{\mathrm{CNC}}$ with a loading of $2.5 \mathrm{wt} \% \mathrm{CNC}$. However, the additional incorporation of $5 \mathrm{wt} \%$ graphitic components led to an increase in interlamellar bridging. A similar bridging effect has been observed for blends of long-chain polymers and clays. ${ }^{29}$ In particular, for the case of CNF, more structural diversity was observed (Fig. S12, ESI $\dagger$ ), including bundling of the fibres and pore filling to occur. Furthermore, no changes in the crystallinity of the microstructure were observed by DSC (Fig. S6, ESI $\dagger$ ) and XRD (Fig. S7, ESI $\dagger$ ).
The orientation-dependent thermal conductivities of $\mathrm{PU}_{\text {pure }}$ and $\mathrm{PU}_{\mathrm{CNC}}$ in vacuum were comparable within the experimental error, which suggests that a small amount of CNCs $(2.5 \mathrm{wt} \%$ loading) is not sufficient to affect the thermal conductivity of PU foams (Fig. 2a). The incorporation of $5 \mathrm{wt} \%$ of CB also did not significantly affect the thermal conductivity for $\mathrm{PU}_{\mathrm{CNC}-\mathrm{CB}}$ in either orientation likely because the CB was present at subpercolating concentrations.

In contrast, $\mathrm{PU}_{\mathrm{CNC}-\mathrm{CNF}}$ samples were significantly more thermally conductive than $\mathrm{PU}_{\text {pure }}$ with $\kappa_{\text {perp }}=59 \pm 5 \mathrm{~mW} \mathrm{~m}^{-1} \mathrm{~K}^{-1}$ and $\kappa_{\text {para }}=158 \pm 11 \mathrm{~mW} \mathrm{~m}^{-1} \mathrm{~K}^{-1}$. The high aspect ratio of CNFs can enable the formation of a percolating network of CNFs in the aligned lamellae. Higher thermal conductivities in $\mathrm{PU}_{\mathrm{CNC}-\mathrm{CNF}}$ samples could be attributed to thermal transport along the graphitic CNF backbone in the lamellae.

For $\mathrm{PU}_{\mathrm{CNC}-\mathrm{CB}}$ and $\mathrm{PU}_{\mathrm{CNC}-\mathrm{CNF}}$, the thermal anisotropy was less pronounced with $\kappa_{\text {para }} / \kappa_{\text {perp }}$ of 2.2 and 2.7 , respectively. As observed from the SEM images (Fig. S5, S11 and S12, ESI $\dagger$ ), the introduction of graphitic materials increased the inter-lamellar bridging and reduced the aspect ratio of the pores. Consequently, more thermal transport in the perpendicular orientation was possible.

The thermal transport behaviour of these composite foams revealed an important contribution of the solid skeleton to realize an effectively isotropic state. The presence of highly conductive CNFs created a mismatch between the thermal conductivities of the lamellae and He such that the thermal anisotropy was maintained at all pressures. In all other cases, the matching thermal conductivity of the He gas $\left(155 \mathrm{~mW} \mathrm{~m}^{-1} \mathrm{~K}^{-1}\right)$ and of the solid PU phase $\left(\sim 200 \mathrm{~mW} \mathrm{~m}^{-1} \mathrm{~K}^{-1}\right)$ allowed these structurally anisotropic foams to behave as an effective single-phase material with an isotropic thermal conductivity (assuming a low thermal interface resistance between the polymer and the gas phase).

All samples show a high degree of reversibility when toggling between anisotropic and isotropic states of thermal conduction (Fig. S14, ESI $\dagger$ ). As was also the case for $\mathrm{PU}_{\text {pure, }}$ the transition between these distinct states occurs on a time scale which is faster than the minimum time resolution of our XFA experiments ( $<$ tens of seconds).

Based on these findings, we derive two design principles for achieving this unique thermal switching behaviour. First, the structural skeleton (solid phase) must provide an anisotropic pathway for thermal energy transport, distinct from that of the gas phase. In this work, this pathway was the percolating network of PU created by the directional growth of the ice crystals during the freeze-casting process. The second criterion is, that the thermal conductivities of the solid and gas phase must match as closely as possible under a particular condition (e.g. pressure, gas composition, and temperature), but be mismatched under another condition.

We also put the thermal transport properties of these foams into the context of other foam and bulk polymer materials using an Ashby plot in Fig. 3c. The thermal conductivity data were acquired in air at atmospheric pressure. Our foams resemble thermal conductivities comparable to standard, isotropic foams (perpendicular orientation), and to soft and highly insulating bulk 

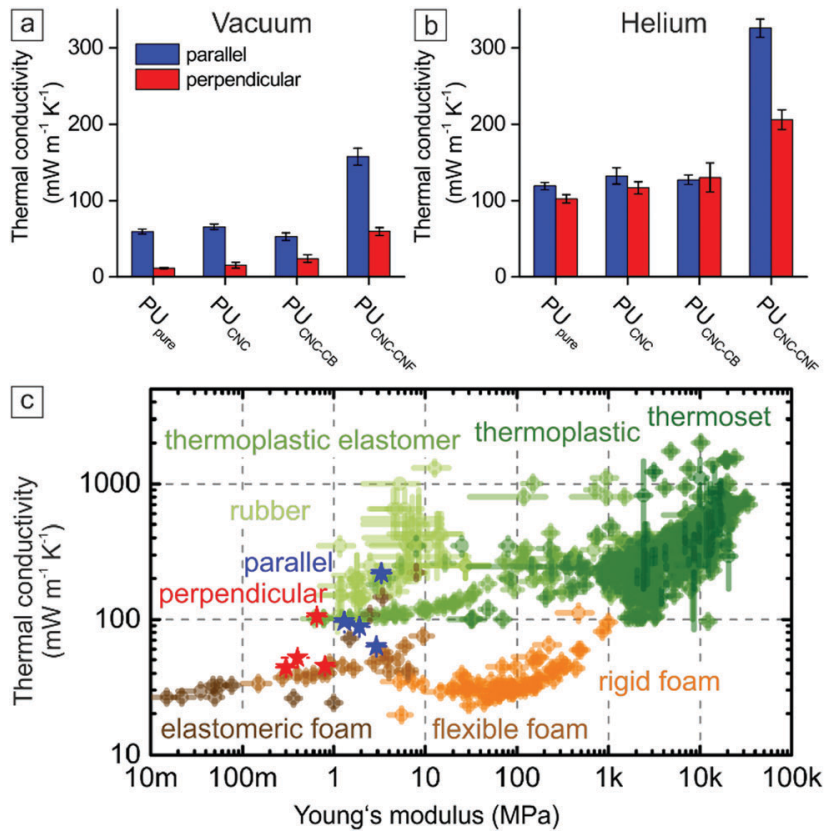

Fig. 3 (a) Thermal conductivities of PU foams with various additives in vacuum. In vacuum, all foams feature an anisotropic thermal conductivity. (b) Thermal conductivity of the same foams measured in He. Only PU $U_{C N C-C N F}$ retains some degree of thermal anisotropy, whereas all other foams feature an isotropic thermal conductivity. (c) Ashby plot relating the thermal conductivity to the mechanical properties of the respective PU foams. The data are in relation to existing polymeric bulk (green) and cellular materials (brown). ${ }^{27}$

polymers (parallel orientation). The $\kappa_{\text {para }}$ values were comparable to those of bulk rubber polymers and thermoplastic elastomers, which typically possess a much higher density $\left(\sim 1000 \mathrm{~kg} \mathrm{~m}^{-3}\right)$ compared to the foam samples under consideration here $\left(210 \mathrm{~kg} \mathrm{~m}^{-3}\right.$ to $300 \mathrm{~kg} \mathrm{~m}^{-3}$ ). The $\kappa_{\text {perp }}$ values were comparable to those of isotropic flexible foams, which typically possess a much lower density $\left(<100 \mathrm{~kg} \mathrm{~m}^{-3}\right)$.

The thermal conductivities of the foams are further correlated with their respective Young's moduli (Fig. 4a). The Young's moduli were determined by compressing the samples up to $50 \%$ strain along the parallel and perpendicular orientations. The influence of additives on the compressive properties of the various PU foams was examined as shown in Fig. 4a. All samples displayed classical deformation behaviour along the parallel orientation, which is characteristic of elastomeric cellular foams (Fig. 4b and Fig. S15, $\mathrm{ESI} \dagger)^{8}{ }^{8}$ The Young's modulus for each sample was determined from the linear elastic region (up to $5 \%$ strain). In all cases, the compressive Young's modulus along the perpendicular orientation was significantly lower than that in the parallel direction. For $\mathrm{PU}_{\text {pure, }}$ the Young's modulus decreased from $1.9 \pm 0.4 \mathrm{MPa}$ (parallel) to $0.302 \pm 0.002 \mathrm{MPa}$ (perpendicular). As illustrated in the Ashby plot (Fig. 3c), the Young's moduli of these PU foams were comparable to those reported for flexible foams, elastomeric foams, and rubber. ${ }^{27}$ The addition of CNCs decreased the Young's modulus along the parallel orientation slightly to $1.3 \pm 0.1 \mathrm{MPa}$, potentially because the CNCs acted as point defects, which allowed for an easier buckling of the lamellae. The addition of CB and CNFs, however, increased the Young's modulus. We attribute this to the reinforcing effects of the stiff graphitic materials. ${ }^{30}$

An advantageous feature of these anisotropic foams is their elasticity. To demonstrate this, the foams were subjected to 20 compression-decompression cycles up to $20 \%$ strain, which was well beyond the onset of buckling in the lamellae for compression in the parallel orientation (Fig. 4b and Fig. S16, ESI $\dagger$ ). The mechanical response was largely reproducible with each cycle. The compression set of the foams was $\sim 8 \%$, with irreversible losses mostly over the first several cycles. Additionally, foams subjected to $50 \%$ compressive strain demonstrated a high recoverability of up to $95 \%$ in the parallel orientation and $71-90 \%$ in the perpendicular orientation (Fig. 4c).

Overall, the mechanical characterization demonstrates that robust PU foams are accessible via freeze-casting of PU dispersions. They show anisotropic compression moduli in the range of typical foam and rubber materials. Most importantly, the foams recover after compression, which renders them suitable for mechanically demanding applications.

\section{Conclusions}

In conclusion, we fabricated a robust and dynamic insulation material via freeze-casting. The material consists of PU foams
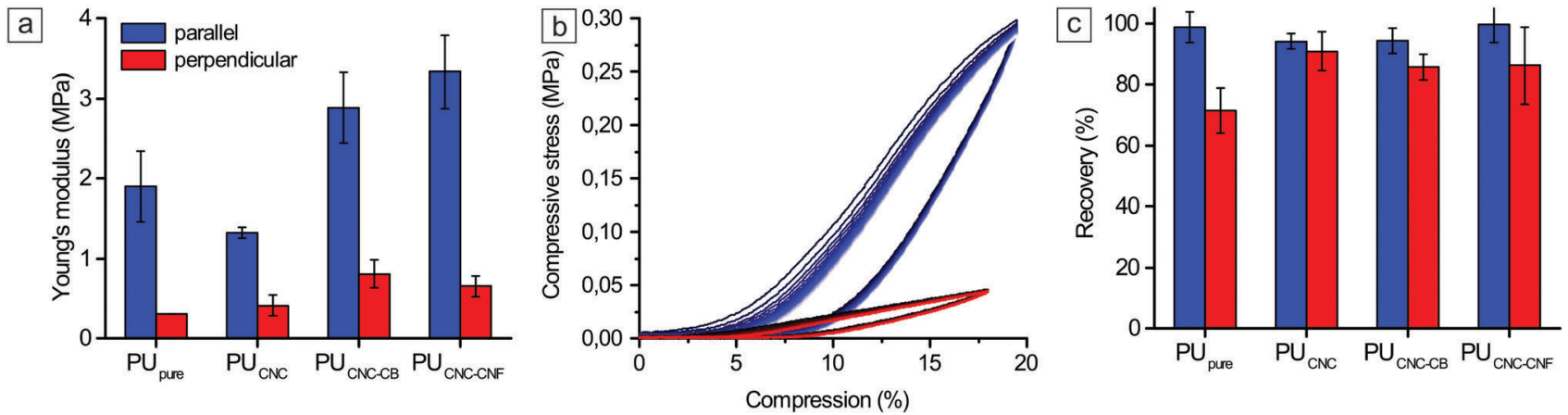

Fig. 4 Mechanical properties of PU foams containing various additives. Blue and red colors describe the data resulting from compression in the parallel and perpendicular orientations, respectively. (a) Orientation-dependent Young's moduli of PU foams. (b) 20 compression cycles up to $20 \%$ compression. (c) Orientation-dependent recovery of the PU foams after $50 \%$ compression. 
with long, oriented lamellae and highly anisotropic pores templated by directional ice growth. Most importantly, we demonstrate the ability of these structurally anisotropic PU foams to transition between isotropic and anisotropic modes of thermal conductivity in a reversible and controllable way. This unique behaviour can be achieved in two distinct ways: by reducing the thermal conductivities of the gas phase via its pressure, and by changing the composition of the gas phase. In order to attain isotropic thermal conduction, the thermal conductivities of the gas and the solid phase need to match as closely as possible. It is important to note that we expect the outlined design principles to be generally applicable to any continuous biphasic material, in which the thermal conductivity of one phase can be altered reversibly. At the same time, these freeze-cast foams are elastic and have Young's moduli comparable to those of standard foam and rubber materials. The anisotropic nature of the skeleton matrix results in a much higher Young's modulus along the parallel compared to the perpendicular orientation. Furthermore, these foams show a high degree of recoverability. Such materials may find application as dynamic insulation materials, ${ }^{4}$ in which both the amplitude and the orientation of thermal conductivity need to be simultaneously controlled. We hope that this work initiates increased efforts towards the generation of cellular materials, in which functionality is derived from the structural anisotropy.

\section{Acknowledgements}

MC thanks NSERC CREATE IDEM for funding. BK thanks the German Academic Scholarship Foundation for funding. XPS spectra were obtained at the Ontario Centre for the Characterization of Advanced Materials (OCCAM) at the University of Toronto. We thank Rana Sodhi for his insight on the XPS characterization. We thank Fabian Nutz for performing DSC measurements, Dr. Markus Drechsler for the cryo-TEM imaging, Jacqueline Uhm for micro-CT, and Dr. Wolfgang Milius for XRD measurements. This project was funded by the Lichtenberg professorship provided by the Volkswagen foundation. Additional support was provided by the SFB 840 from the German Research Foundation.

\section{Notes and references}

1 R. Zheng, J. Gao, J. Wang and G. Chen, Nat. Commun., 2011, 2, 289.

2 T. Zhang and T. Luo, ACS Nano, 2013, 7, 7592-7600.

3 M. J. Martinez-Perez, A. Fornieri and F. Giazotto, Nat. Nanotechnol., 2015, 10, 303-307.

4 J. Lee, V. Varshney, A. K. Roy, J. B. Ferguson and B. L. Farmer, Nano Lett., 2012, 12, 3491-3496.
5 B. Wicklein, A. Kocjan, G. Salazar-Alvarez, F. Carosio, G. Camino, M. Antonietti and L. Bergstrom, Nat. Nanotechnol., 2015, 10, 277-283.

6 H. X. Zheng, H. R. Shan, Y. Bai, X. F. Wang, L. F. Liu, J. Y. Yu and B. Ding, RSC Adv., 2015, 5, 91813-91820.

7 B. P. Jelle, Energy and Buildings, 2011, 43, 2549-2563.

8 L. J. Gibson and M. F. Ashby, Cellular Solids: Structure and Properties, Cambridge University Press, Cambridge, UK, 2nd edn, 1999.

9 T.-Y. Wei, S.-Y. Lu and Y.-C. Chang, J. Phys. Chem. C, 2009, 113, 7424-7428.

10 D. M. Smith, A. Maskara and U. Boes, J. Non-Cryst. Solids, 1998, 225, 254-259.

11 N. Hüsing and U. Schubert, Angew. Chem., 1998, 37, 22-45.

12 K. E. Parmenter and F. Milstein, J. Non-Cryst. Solids, 1998, 223, 179-189.

13 S.-T. Lee, C. B. Park and N. S. Ramesh, Polymeric Foams: Science and Technology, Taylor \& Francis, Boca Raton, 2007.

14 S. Liu, J. Duvigneau and G. J. Vancso, Eur. Polym. J., 2015, 65, 33-45.

15 B. Notario, J. Pinto, E. Solorzano, J. A. de Saja, M. Dumon and M. A. Rodríguez-Pérez, Polymer, 2015, 56, 57-67.

16 S. Deville, J. Mater. Res., 2013, 28, 2202-2219.

17 H. Bai, Y. Chen, B. Delattre, A. P. Tomsia and R. O. Ritchie, Sci. Adv., 2015, 1, e1500849.

18 S. Deville, E. Saiz, R. K. Nalla and A. P. Tomsia, Science, 2006, 311, 515-518.

19 L. L. da Silva and F. Galembeck, J. Mater. Chem. A, 2015, 3, 7263-7272.

20 S. Deville, E. Maire, G. Bernard-Granger, A. Lasalle, A. Bogner, C. Gauthier, J. Leloup and C. Guizard, Nat. Mater., 2009, 8, 966-972.

21 S. R. Hostler, A. R. Abramson, M. D. Gawryla, S. A. Bandi and D. A. Schiraldi, Int. J. Heat Mass Transfer, 2009, 52, 665-669.

22 S. Benli, Ü. Yilmazer, F. Pekel and S. Özkar, J. Appl. Polym. Sci., 1998, 68, 1057-1065.

23 N. Song, D. Jiao, P. Ding, S. Cui, S. Tang and L. Shi, J. Mater. Chem. C, 2016, 4, 305-314.

24 M. Knudsen, Ann. Phys., 1911, 339, 593-656.

25 E. W. Lemmon and R. T. Jacobsen, Int. J. Thermophys., 2004, 25, 21-69.

26 S. N. Schiffres, K. H. Kim, L. Hu, A. J. H. McGaughey, M. F. Islam and J. A. Malen, Adv. Funct. Mater., 2012, 22, 5251-5258.

27 GRANTA, CES selector 2015, Granta Design Limited, Cambridge, UK, 2015.

28 C. Olivier, C. Moreau, P. Bertoncini, H. Bizot, O. Chauvet and B. Cathala, Langmuir, 2012, 28, 12463-12471.

29 M. D. Gawryla, D. A. Schiraldi, MRS Proceedings, 2011, 1188LL06-07.

30 M. Antunes and J. I. Velasco, Prog. Polym. Sci., 2014, 39, 486-509. 